\title{
Maximal strength training as physical rehabilitation for patients with substance use disorder; a randomized controlled trial
}

\author{
Runar Unhjem ${ }^{1 *}$, Grete Flemmen ${ }^{1,2}$, Jan Hoff ${ }^{1,3}$ and Eivind Wang ${ }^{1,4,5}$
}

\begin{abstract}
Background: Patients with substance use disorder (SUD) suffer from multiple health and psychosocial problems. Because poor physical capacities following an inactive lifestyle may indeed contribute to these problems, physical training is often suggested as an attractive supplement to conventional SUD treatment. Strength training is shown to increase muscle strength and effectively improve health and longevity. Therefore we investigated the feasibility and effect of a maximal strength training intervention for SUD patients in clinical treatment.

Methods: 16 males and 8 females were randomized into a training group (TG) and a control group (CG). The TG performed lower extremities maximal strength training (85-90 \% of 1 repetition maximum (1RM)) 3 times a week for 8 weeks, while the CG participated in conventional clinical activities.

Results: The TG increased hack squat 1RM (88 $\pm 54 \%)$, plantar flexion 1RM (26 $\pm 20 \%)$, hack squat rate of force development (82 $\pm 29 \%)$ and peak force $(11 \pm 5 \%)$. Additionally, the TG improved neural function, expressed as voluntary V-wave (88 $\pm 83 \%)$. The CG displayed no change in any physical parameters. The TG also reduced anxiety and insomnia, while the $C G$ reduced anxiety.

Conclusion: Maximal strength training was feasible for SUD patients in treatment, and improved multiple risk factors for falls, fractures and lifestyle related diseases. As conventional treatment appears to have no effect on muscle strength, systematic strength training should be implemented as part of clinical practice.
\end{abstract}

Trial registration: ClinicalTrials.gov Identifier: NCT02218970 (August 14, 2014).

Keywords: Muscle strength, One repetition maximum, Rate of force development, V-wave, Physical health, Mental health

\section{Background}

In addition to their drug abuse, patients with substance use disorder (SUD) suffer from multiple health and psychosocial comorbidities, resulting in a life expectancy 20-30 years less than the general population $[1,2]$. Compared to the average population these patients are more frequently represented in medical care, with an elevated incidence of cardiovascular disease $[1,2]$, diabetes $[1,2]$, cancer $[1,2]$, suicide [1,2], as well as traumas, falls and fractures [3-5]. Recent findings in our laboratory show that muscle

\footnotetext{
* Correspondence: Runar.Unhjem@gmail.com

${ }^{1}$ Department of Circulation and Medical imaging, Faculty of Medicine, the Norwegian University of Science and Technology, Prinsesse Kristinas gt. 3, 7006 Trondheim, Norway

Full list of author information is available at the end of the article
}

strength and aerobic fitness are markedly reduced in SUD patients compared to healthy age-matched individuals [6]. Low muscle strength is associated with increased incidence of falls and fractures [7, 8], poor mechanical efficiency [9], elevated risk of cancer [10] and cardiovascular disease [11], and is even shown to be an independent predictor of allcause mortality in both patient populations and healthy [12-14].

Strength training has become an increasingly common measure to improve muscle strength in different patient populations, and effectively reduce the risk of medical conditions and mortality. Maximal strength training, with heavy loads ( $>85 \%$ of 1 repetition maximum $(1 R M))$ and emphasis on intended concentric velocity has been successfully applied in multiple patient 
populations in our labs, and is shown to induce particularly large improvements in rate of force development (RFD) and muscle strength [15-19]. The improvements in maximal strength and RFD are suggested to predominantly rely on neural factors, with little or no change in body mass $[9,18,20]$, which results in the training being even more suitable in populations where gains in weight are not sought after. Importantly, no injuries have been reported following these interventions, indicating that the training is not only effective, but also safe. Perhaps even more than the maximal strength, rapid force development is shown to be important for functional status, mechanical efficiency, balance adjustments and the prevention of falls and fractures [21-23]. Because the RFD relies mainly on neuromuscular properties [24], strength training applied to induce functional gain in patient populations should target neural adaptations. Assessed by the use of evoked reflex recordings, our research group has previously documented neural adaptations in both patient and healthy populations following maximal strength training $[17,25]$.

Although SUD patients are reported to have low muscle strength and aerobic capacity [6], there are few studies of systematic physical training as a part of clinical SUD treatment [26]. While physical activity is commonly used in conventional treatment [27], it appears not to apply a sufficient overload for taxing the muscular strength. Thus, maximal strength training would likely offer additional health benefits, and effectively reduce the risk of medical conditions. In addition to physical benefits, strength training is shown to have a positive effect on mental health, reducing anxiety and depression levels [28-30]. A low muscle strength has even been shown to independently be associated with an elevated rate of suicide [31]. In general, adherence to an exercise regime is also suggested to improve treatment outcomes and possibly reduce relapse rates in patients suffering from alcohol and substance abuse $[26,32]$.

Since physical activity in clinical treatment often appear random and unstructured [33], without the sufficient overload to produce gains in muscular strength, the aim of this study was to assess if a maximal strength training intervention was feasible for SUD patients, and would yield the previously documented beneficial physical and mental effects of such a training regime. We hypothesized that (1) SUD patients would be able to carry out the 8 week maximal strength training intervention, and (2) that the training group would improve maximal strength, RFD, efferent neural drive, depression, anxiety and insomnia more than the control group that participated in conventional treatment.

\section{Methods \\ Subjects}

24 patients diagnosed with SUD, classified within ICD10: F10-F19 (mental and behavioral disorders due to psychoactive substance use), were included in the study from February to March 2013. All subjects participated in a $\sim 3$ month residential long term treatment at a substance abuse clinic at the University hospital, and had amphetamine as their primary drug. After providing their informed consents subjects were randomized to either a maximal strength training group (TG) or a control group (CG) participating in conventional activities (Fig. 1). Subjects were assigned a number between 1 and 24 , and randomization was performed using a publicly accessible official website designed for research randomization (https://www.randomizer.org). Subjects were excluded if they had been abstinent and/or systematically participated in strength training for the last six months. Other exclusion criteria were cardiovascular or respiratory disease, not being able to carry out the testing procedure or failure to participate in at least 20/24 training sessions. Patient characteristics and medical use are shown in Table 1. The study was approved by the regional ethical committee (REK-nord) and conducted in accordance with the declaration of Helsinki.

\section{Extent of drug use}

To get an overview of the extent of drug use the first page of EuropASI was applied [34]. The index quantifies which substances the subject has used, age at first time drug use and years of use. Further the clinic provided information of prescribed medicine for the overall participating group of patients. Patient characteristics and medical use are given in Table 1.

\section{Testing procedure}

All subjects conducted the testing procedure before and after the 8 week training intervention. On the day of testing, neuromuscular measurements (V-wave) were carried out first, followed by 1RM hack squat, 1RM plantar flexion and hack squat RFD. After the strength measurements, psychological questionnaires were filled out to assess levels of insomnia, anxiety and depression. Subjects were asked to not engage in any physical training on the day of testing or the day before.

\section{Strength measurements}

One repetition maximum (1RM) was measured in hack squat and plantar flexion. Hack squat 1RM was obtained in a hack squat machine (Impulse Fitness IT7006, Shandong, China) angled $45^{\circ}$ to vertical. For the plantar flexion test, the participants were seated in a calf rise machine (Impulse Health Tech IT7005, Shandong, China), 


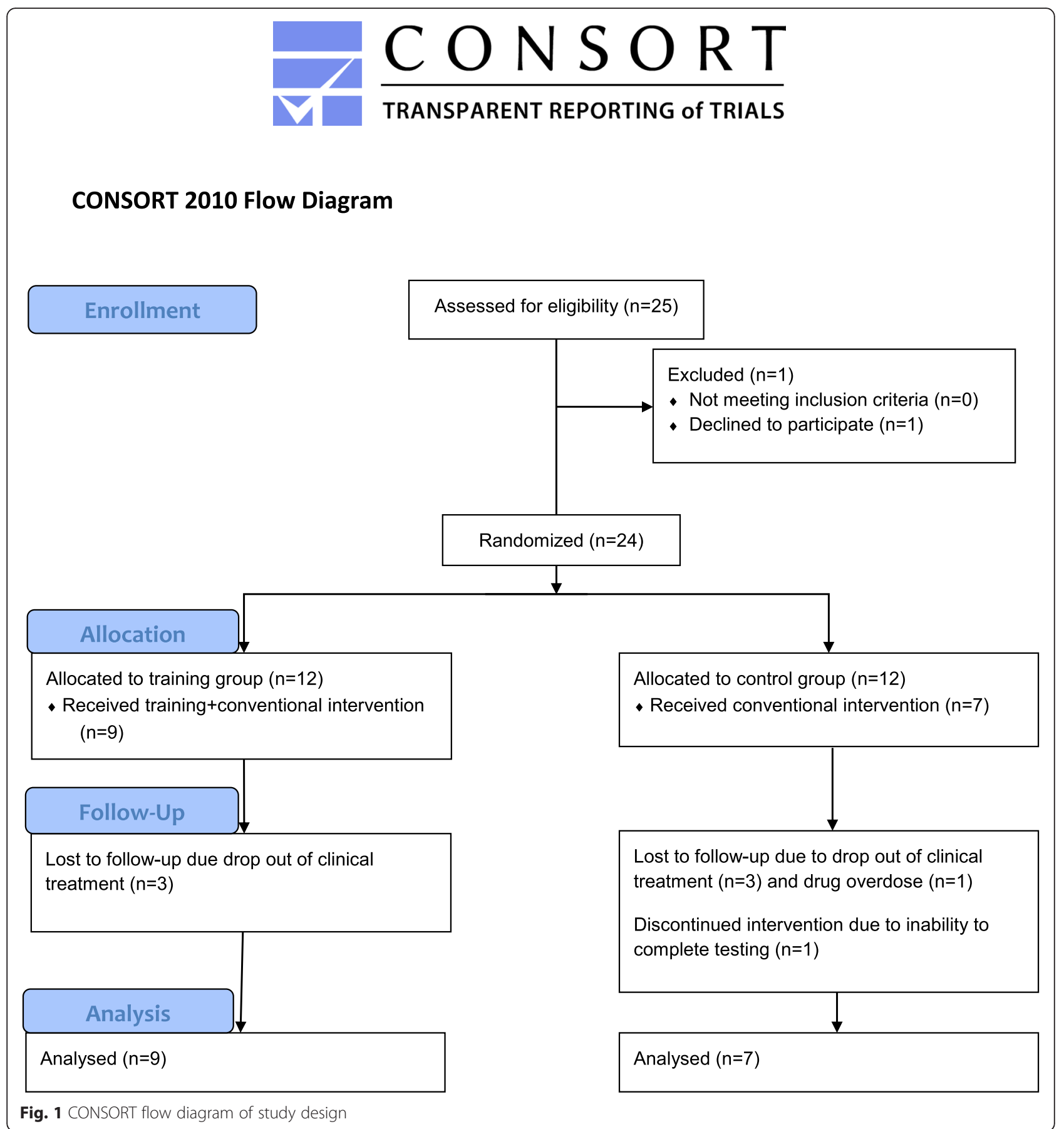

with a knee joint angle of $\sim 90^{\circ}$, and performed their lifts from an ankle joint angle of $\sim 20^{\circ}$ dorsiflexion in the lower position, up to $\sim 30^{\circ}$ plantar flexion in the upper position. Before testing the subjects were familiarized with the testing apparatus during an extensive warm up procedure, however no additional familiarization session was arranged. For both hack squat and plantar flexion, 1RM was achieved by increasing the load by $5-10 \mathrm{~kg}$ until the subject was not able to complete the lift. A three minutes rest was given between each trial, and correct joint angles were ensured. 1RM was achieved within 6-9 trials, and the highest load completed was recorded as 1RM.

RFD was recorded in the hack squat machine with a force platform at $2000 \mathrm{~Hz}$ (9286AA, Kistler, Switzerland) attached to the foot plate. Each subject was given three attempts with a load corresponding to $80 \%$ of pretest $1 R M$. Only the best trial was used for analyzes. The subjects were instructed to move slowly down to a knee 
Table 1 Patient characteristics and medical use

\begin{tabular}{|c|c|c|c|}
\hline & $\begin{array}{l}\text { TG } \\
(n=9)\end{array}$ & $\begin{array}{l}\text { CG } \\
(n=7)\end{array}$ & $\begin{array}{l}\text { Combined } \\
(n=16) \\
\end{array}$ \\
\hline Men/Women (n) & $6 / 3$ & $7 / 0$ & $13 / 3$ \\
\hline Age (yr) & $33 \pm 9$ & $29 \pm 5$ & $32 \pm 8$ \\
\hline Weight (kg) & $80.2 \pm 18.2$ & $81.8 \pm 9.6$ & $80.9 \pm 14.3$ \\
\hline Height (cm) & $173 \pm 10$ & $181 \pm 5$ & $177 \pm 9$ \\
\hline First time drug use (age) & $14 \pm 2$ & $15 \pm 2$ & $14 \pm 2$ \\
\hline Duration of abuse (yr) & $13 \pm 10$ & $11 \pm 4$ & $12 \pm 8$ \\
\hline Current Smoker & 7 & 6 & 13 \\
\hline \multicolumn{4}{|l|}{ Primary drug: } \\
\hline Amphetamine & 9 & 7 & 16 \\
\hline \multicolumn{4}{|l|}{ Secondary drug: } \\
\hline Alcohol & 4 & 1 & 5 \\
\hline Cocaine & & 1 & 1 \\
\hline Cannabis & 5 & 5 & 10 \\
\hline \multicolumn{4}{|c|}{ Symptoms for medicine prescription: } \\
\hline ADHD & 1 & 1 & 2 \\
\hline Allergies & 3 & 4 & 7 \\
\hline Anxiety & & 3 & 3 \\
\hline Arthiritis & 2 & & 2 \\
\hline Asthma/COPD & 3 & 1 & 4 \\
\hline Depression & 3 & 1 & 4 \\
\hline Epilepsy & & 1 & 1 \\
\hline Hypertension & 5 & & 5 \\
\hline Schizofenia/Bipolar & 4 & 1 & 5 \\
\hline Migrene & 3 & & 3 \\
\hline Substitutional treatment & & 1 & 1 \\
\hline Other & 5 & 1 & 6 \\
\hline
\end{tabular}

Data are presented as mean $\pm S D, T G$; training group, $C G$; control group. Type of medication is reported on indication of symptoms according to common directory. The prescribed medicine in substitutional treatment is subuxone. Others: atherothrombosis, diabetes, infections

joint angle of $90^{\circ}$, have a short stop to avoid eccentric action involvement, and then mobilize maximally in the concentric phase of the movement. Three minutes rest was given between each trial. The highest concentric force was recorded as peak force and RFD was calculated as $\Delta$ force between $10 \%$ and $90 \%$ of peak force [9].

\section{Neuromuscular measurements}

Neuromuscular measurements were assessed by voluntary $\mathrm{V}$-waves, with the subjects seated in a fixed version of the plantar flexion apparatus used for dynamic strength measurements. The $\mathrm{V}$-wave method involves electrical stimulation of the tibial nerve, applied to evoke reflex potentials and motor potentials in afferent and efferent nerves. During supramaximal electrical stimulations all afferent and efferent nerve fibers are recruited simultaneously, and the reflex volley traveling the muscle spindle reflex circuit will collide with electrically evoked action potentials traveling antidromically in the efferent axons. Because of these collisions the reflex volley will be completely abolished during rest and not reach the muscle. In contrast, during maximal voluntary contraction (MVC) the efferent drive to the muscle will collide with the antidromic potentials, leaving some efferent axons open for transmission of the reflex. A higher efferent drive will clear more axons for reflex transmission, and will thus allow more of the reflex volley to pass through to the muscle, where it is recorded as a V-wave. Based on this, the amplitude of $\mathrm{V}$-wave is used to express the efferent neural drive during MVC.

Reflex potentials were evoked by a current stimulator (DS7AH, Digitimer, Welwyn Garden City, UK), in the tibial nerve, in the popliteal fossa. The electrical current was delivered by gel-coated (Lectron 2 conductive gel, Pharmaceutical innovations INC, Newark, NJ, USA) bipolar felt pad electrodes, $25 \mathrm{~mm}$ between tips, $8 \mathrm{~mm}$ diameter (Digitimer, Welwyn Garden City, UK). The electrodes were held by hand throughout the testing procedure, and positioned at the site evoking the largest reflex amplitude. Evoked potentials were recorded through self-adhesive AG/AgCI electrodes (Ambu, M00-S/50, Ballerup, Denmark) placed as recommended by SENIAM [35] on m. soleus. Before electrode attachment the skin was carefully preparated to minimize the interelectrode impedance; impedance level $<5 \mathrm{k} \Omega$ were required. To provide equal conditions from pre- to posttest, pictures were taken of the electrode placement at pretest, and used for identical positioning at posttest.

Searching for the maximal direct motor potential $\left(M_{\max }\right)$ the current intensity was gradually increased by 2-5 mA until the M-wave reached a plateau. Between 70 and $180 \mathrm{~mA}$ was needed to evoke $\mathrm{M}_{\max }$. To validate the $\mathrm{M}_{\max }$ three supramaximal stimuli at $150 \%$ of the current intensity needed to reach the plateau were given. Eight V-waves were evoked during MVC by delivering a supramaximal (150\%) stimulus at the point where the subject reached $\sim 90 \%$ of MVC force. Each MVC was separated by $1 \mathrm{~min}$ rest. Only $\mathrm{V}$-wave recordings, in which the $M$-wave was $>90 \%$ of $M_{\max }$, were used for analyzes. The maximal $\mathrm{V}$-wave amplitude $\left(\mathrm{V}_{\max }\right)$ was expressed relative to $M_{\max }(\mathrm{V} / \mathrm{M}$-ratio), to allow between subjects comparisons. Changes in V/M-ratio are used to express changes in efferent drive following training.

\section{Psychological questionnaires}

In addition to the physical testing two questionnaires were implemented; Insomnia Severity Index (ISI) to measure level of insomnia, and Hospital Anxiety \& Depression Scale (HAD), used to estimate symptoms of anxiety and depression. These self-report questionnaires were answered in conjunction with the pre- and posttest 
of muscular strength, as measures of psychological changes during the period of the study. The ISI has been evaluated to be a clinically useful tool for screening and quantifying perceived insomnia severity [36]. It is composed of 7 items targeting different categories of sleep disturbance severity. The items are rated at a five-point Likert scale (0-4) summed up to provide a total score ranging from $0-28$, where a higher score indicates more severe insomnia. The score categories are 0-7 (no clinically significant insomnia), 814 (subthreshold insomnia), 15-21 (clinical insomnia, moderate severity) and 22-28 (clinical insomnia, severe). The HAD self-assessment scale consists of a fourteen item scale, seven items relate to anxiety and seven relate to depression. On the seven item HADS subscales a score of 0-7 for either subscale is estimated within the normal range, a score of 11 or higher implies a probable presence of a mood disorder. A score of $8-10$ is considered signs of a mood disorder [37].

\section{Training intervention}

Both the TG and the CG attended the regular treatment program at the substance abuse clinic during the intervention period. The treatment program activities included: Ballgames (indoor-soccer, bandy and volleyball), yoga, stretching, outdoor walking, low resistance strength training (estimated $<50 \%$ of $1 \mathrm{RM}$ ), ceramics, TV games and card games. Together this resulted in a total of $\sim 3 \mathrm{~h}$ of physical activity per week. In addition, the TG received maximal strength training 3 times a week for a period of 8 weeks. The training intervention consisted of two exercises; hack squat and plantar flexion. Both exercises consisted of 4 sets of 4-5 repetitions, corresponding to $85-90 \%$ of $1 \mathrm{RM}$. The training load was increased with $5 \mathrm{~kg}$ if 5 repetitions were accomplished in the last set. Both exercises were conducted with a slow controlled movement in the eccentric phase, a short stop, and then maximal mobilization of force in the concentric movement. Hack squat was performed with $90^{\circ}$ knee joint angle, while the plantar flexion exercise was performed from an ankle joint angle of $\sim 20^{\circ}$ dorsiflexion up to $\sim 30^{\circ}$ plantar flexion. Every training session was supervised to ensure proper technique and progression throughout the training period. While the TG participated in the supervised strength training, the CG chose to participate in selfelected supervised activities among the offered sports or games in the clinical treatment program.

\section{Statistical analyzes}

Statistical analyzes were done using IBM SPSS Statistics 21 (Chicago, IL, USA), while figures were created using GraphPad Prism 5 (San Diego, USA). Independent and paired $t$ tests were used to examine differences between groups at baseline and within groups following training, respectively. Between group differences following training were determined by use of two-way repeated ANOVAS. The Pearson test for linear regression was applied to assess correlations. Statistical significance level was set to $p<0.05$. All variables exhibited normal distribution, as confirmed by quantile-quantile plots. Data are presented as mean \pm SD unless otherwise noted.

\section{Results \\ Completion}

Of the 24 patients that were included in the study, 16 subjects completed the study period. 3 patients in the TG dropped out of the clinical treatment, and hence also dropped out of the study. In the CG 5 subjects dropped out; 3 patients dropped out of clinical treatment, 1 patient were not able to complete the testing procedure and 1 patient died from drug overdose. The withdrawal in the two groups resulted in an uneven distribution of genders, leaving no females in the CG at posttest. The participants in the TG adhered to $23 \pm 1$ of the 24 scheduled training sessions during the training period. The patients completed all commenced training sessions and the targeted intensity (85-90\% of 1RM) was reached in all sessions.

\section{Muscle strength measurements}

For the 16 subjects that completed the study, there was no significant difference between the TG and the CG in any of the measured strength parameters at pretest. After 8 weeks of maximal strength training the TG increased 1 RM hack squat by $88 \pm 54 \%(p<0.01)$ (Fig. 2 ), whereas plantar flexion $1 \mathrm{RM}$ increased from $98 \pm 23 \mathrm{~kg}$ to $121 \pm 17 \mathrm{~kg}(26 \pm 20 \%, p<0.01)$. The TG also increased RFD by $82 \pm 28 \%(p<0.01)$ (Fig. 3$)$, whereas peak force increased from $1846 \pm 357 \mathrm{~N}$ to $2045 \pm 415 \mathrm{~N}$ $(11 \pm 5 \%, p<0.01)$. No significant changes were observed in the CG for any of the strength parameters.

\section{Neuromuscular measurements}

Maximal strength training led to an enhanced efferent neural drive in the TG. Following the 8 week training intervention the TG increased $\mathrm{m}$. soleus $\mathrm{V}_{\max }$ from $1583 \pm 1596 \mu \mathrm{v}$ to $2189 \pm 1375 \mu \mathrm{v}(92 \pm 95 \%(p<0.01))$. As there was no observed change in $m$. soleus $M_{\max }$ $(6379 \pm 2188 \mu \mathrm{v}$ vs. $6332 \pm 2244 \mu \mathrm{v})$, this resulted in an $88 \pm 83 \%(p<0.01)$ increase in $\mathrm{m}$. soleus $\mathrm{V} / \mathrm{M}$-ratio (Fig. 4). No significant changes were observed for the CG. Finally, $\Delta \mathrm{V} / \mathrm{M}$-ratio correlated with $\Delta$ hack squat $1 \mathrm{RM}(\mathrm{r}=0.44, p<0.05)$ and $\Delta$ plantar flexion 1RM $(\mathrm{r}=0.57, p<0.05)$. 


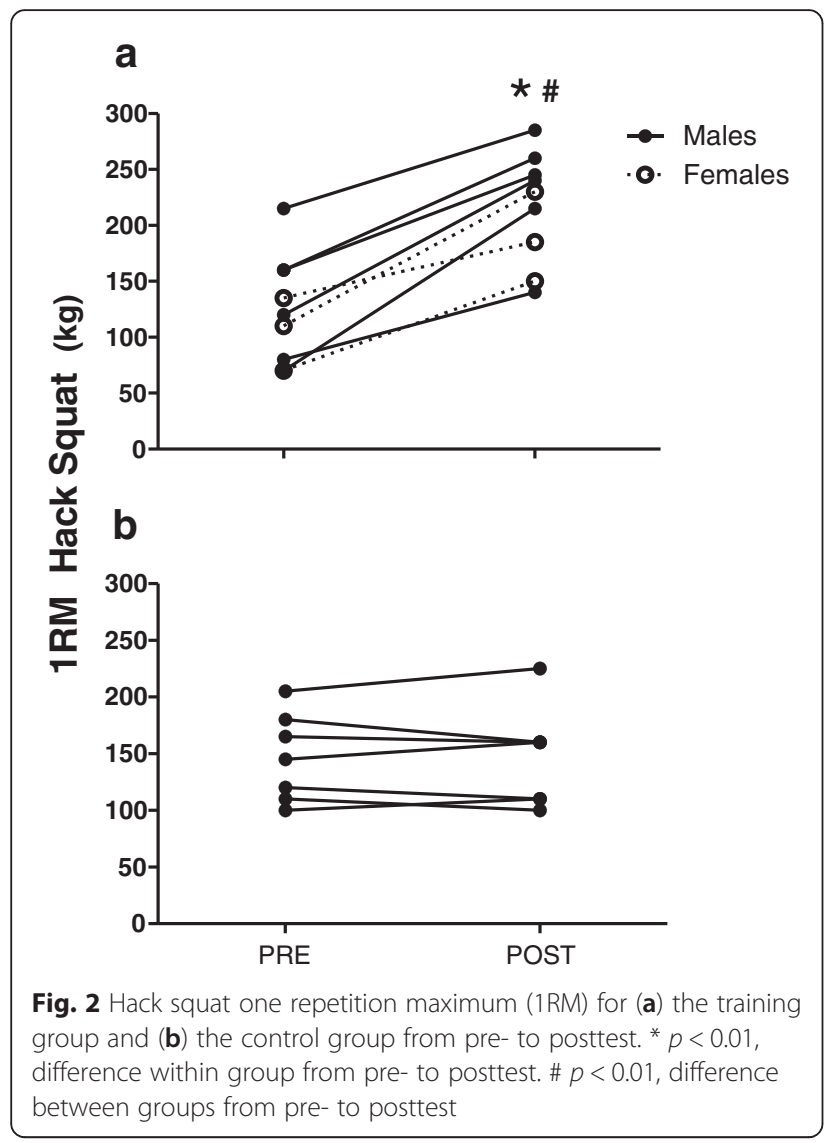

\section{Psychosocial variables}

Both the TG and the CG scored within "probable presence of mood disorder" at inclusion, with elevated scores of anxiety and insomnia. Following the study period both the TG and the CG displayed significant within group reductions in anxiety level $(p<0.05)$, while the level of insomnia significantly decreased only in the TG $(p<0.05)$ (Table 2$)$. Also the level of depression tended to decrease in both groups ( $p=0.11$ for the TG and $p=0.10$ for the CG). Neither of the within group differences were apparent as between-group differences.

\section{Discussion}

\section{Main findings}

SUD patients suffer from physical and psychological deconditioning as a consequence of their detrimental lifestyle. Since strength training is documented to improve both physical and mental health, we sought to investigate the feasibility and efficiency of a maximal strength training regime for a group of SUD patients in residential treatment. The main findings were that 1 ) A maximal strength training intervention was feasible for SUD patients in treatment, 2) Maximal strength training effectively improved maximal strength and muscle force development characteristics, likely caused by alterations

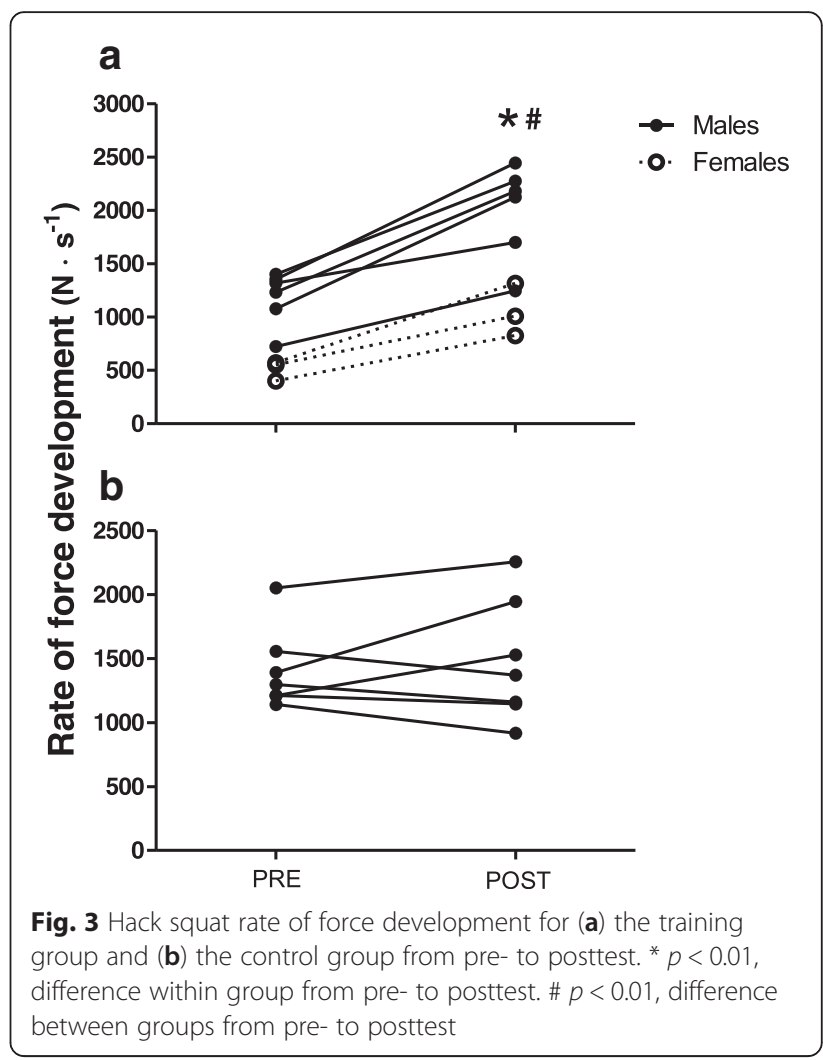

in the central nervous system, 3) Anxiety and insomnia were improved following the clinical treatment period.

Improved maximal strength and muscle force development characteristics

As expected the SUD patients that completed the strength training intervention displayed large improvements in all the measured strength parameters. The $88 \%$ increase in hack squat 1 RM after 8 weeks of

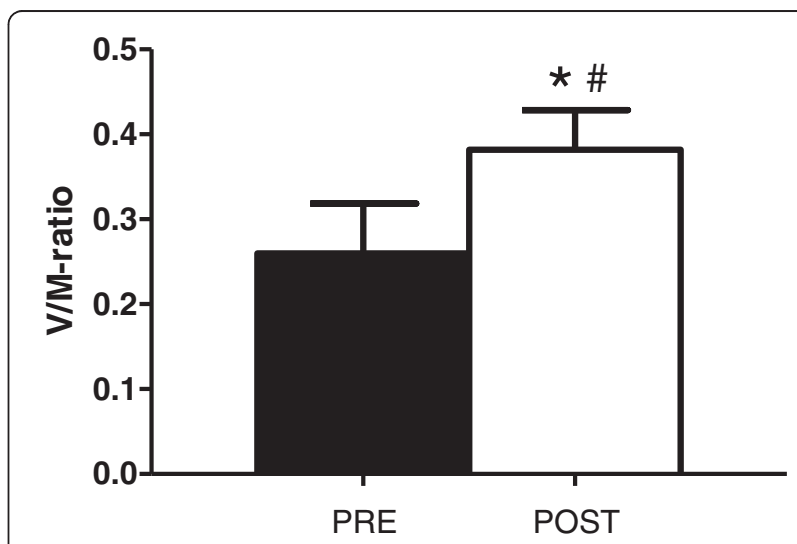

Fig. 4 Data are presented as mean \pm SE. Maximal $V$-wave/maximal M-wave (V/M-ratio) for the training group at pre- and posttest. * $p<0.01$, difference within group from pre- to posttest. \# $p<0.01$, different from the control group from pre- to posttest 
Table 2 Psychological measurements, changes from pre- to posttest (scores from insomnia severity index and hospital anxiety and depression scale questionnaires)

\begin{tabular}{lllll}
\hline & TG $(n=9)$ & \multicolumn{3}{c}{ CG $(n=7)$} \\
\hline & Pre & Post & Pre & Post \\
Anxiety (0-21) & $12.3 \pm 5.8$ & $6.3 \pm 3.9 *$ & $11.1 \pm 4.5$ & $8.0 \pm 4.8 *$ \\
Depression (0-21) & $5.2 \pm 2.1$ & $3.0 \pm 1.6$ & $7.4 \pm 4.7$ & $4.9 \pm 3.8$ \\
Insomnia (0-28) & $9.2 \pm 6.5$ & $3.0 \pm 2.0 *$ & $13.3 \pm 6.2$ & $10.1 \pm 5.3$
\end{tabular}

Data are presented as mean $\pm S D, T G$; Training group, $C G$; Control group. Score categories anxiety and depression: Normal (0-7); signs of mood disorder (8-10); probable presence of mood disorder (11-21). Score categories insomnia: No clinically significant insomnia (0-7); subthreshold insomnia (8-14); clinical insomnia, moderate severity (15-21); clinical insomnia, severe $(22-28) .{ }^{*} p<0.05$, difference within group pre- to posttest

training is even somewhat higher than most previous maximal strength training studies, typically ranging between 25-45 \% [9, 18, 19, 38, 39]. Since familiarization was included as a part of the training intervention in this study, this likely contributed to the large strength gain. Additionally, the very low baseline of the weakest subject, which allowed for a very large percentage improvement of $\sim 200 \%$, also contributed to the high percentage improvement of the TG. Nevertheless, our findings demonstrate the large strength gain achievable when heavy loads and maximal intended concentric velocity are emphasized in strength training. Recognizing that the low physical baseline of the patients in the current study allows large training adaptations, both physiologically and mathematically, the large increase in hack squat 1RM highlights the clinical benefit of a high intensity training intervention in effective physical rehabilitation. The health benefits from an $88 \%$ improvement in leg muscle strength are unquestionable. Ortega et al. [31] reported that Swedish men with high muscular strength had $35 \%$ lower risk of developing cardiovascular disease, 15-65\% lower risk of having any psychiatric diagnosis and $20 \%$ lower risk of all cause mortality when compared to men with low muscle strength. Also Ruiz et al. [10,12] found the risk of mortality from cancer, cardiovascular disease and other causes to be inversely correlated with muscle strength. Both the Ortega et al. (2012) study and the Ruiz et al. [10, 12] studies emphasize that subjects with low and very low muscle strength particularly suffer an increased risk of medical complications. Considering this, increasing the strength of the weakest individuals would provide the largest health benefit. Although we did not compare our subjects with a reference group some of the patients in the current study stood out as particularly weak. Interestingly it was these patients who apparently seemed to benefit the most from the training. This visual observation was also reflected in the psychosocial questionnaires, where the three weakest subjects exhibited substantial improvements in the psychosocial variables following the training period.
High muscle strength is also associated with lower risk of falls and fractures [7, 8]. Moreland et al. [40] reported that subjects with low and very low muscle strength exhibited elevated risk of single and recurrent falling (Odds ratio: 1.31-5.06). Considering the high incidence of non-drug related hospitalizations among SUDpatients, typically including traumas, falls and fractures [3-5], it is likely that the improved muscle strength would have a preventive effect on these high injury- and hospitalization rates. Balance adjustments and fall prevention do not only require maximal strength; the ability of rapid muscle contractions is often just as important, since the time frame to avoid a fall is short [21, 22, 41]. Because strength training with heavy loads and maximal concentric mobilization is associated with large gain in explosive strength, maximal strength training is argued to be particularly beneficial to induce gain in motor function. The $82 \%$ increase in RFD in the current study adds evidence of the large improvements in explosive strength following maximal strength training regimes, and is similar to previous reports from our research group $[18,38,39]$.

\section{Neuromuscular alterations and maximal strength training} The $\sim$ twofold increase in V/M-ratio highlights that neuromuscular changes largely contributed to the gain in muscle strength. Although there is agreement that training-induced changes in muscle strength relies on a combination of neuromuscular and anabolic adaptations [42], studies involving maximal strength training have often claimed that the improvements were mainly of neuromuscular origin, due to large improvements in 1RM and RFD, with no change in body weight $[9,18,20]$. Based on the comparable large improvements in V/M-ratio and maximal strength, as well as the lack of change in body weight, our findings are in line with this notion. It is unlikely that the low number of repetitions, and thus low anabolic effect, was sufficient to induce any significant muscle growth, while the heavy loads and maximal mobilization seems to be optimal for neural adaptations $[20,43]$. The $88 \%$ increase in V/M-ratio is slightly higher compared to other strength training studies, typically displaying improvements of 50-80 \% [44, 45]. However, these interventions have been conducted with a lower training intensity than the current, consequently also resulting in smaller improvements maximal strength. Therefore, in combination, our findings and previous studies, exhibits corresponding improvements in neuromuscular adaptations and muscular strength. Specifically, the changes in $\mathrm{V}$-wave amplitude in the current study likely reflects an enhanced efferent neural drive to the muscle, probably due to increased motor unit firing frequency and/or increased motoneuron recruitment $[44,46]$. This is because a higher efferent drive would allow more of the electrically 
evoked reflex volley to pass through to the muscle, hence resulting in the increased amplitude of the $\mathrm{V}$-wave.

\section{Feasibility of maximal strength training in substance use clinical treatment}

The $75 \%$ completion rate of the TG in the current study exemplifies that although maximal strength training may be considered strenuous, SUD patients are in general capable of engaging in physically demanding training regimes. To date there have been few studies examining intensive physical training in SUD patients, but we have recently shown that also intensive endurance training is feasible for this patient group [33]. In agreement with our findings from the endurance training study, the SUD patients reported no difficulties carrying out the strength training, and the targeted intensity (85-90\% of $1 \mathrm{RM}$ ) was reached in all commenced training sessions, without any reports of pain or discomfort. Importantly, most of the subjects that participated reported that they found the simple and robust training motivating, and that they enjoyed observing their own steady and impressing large progression throughout the study. Although SUD is commonly associated with high rates of nonattendance and relapse [47, 48], we experienced no issues regarding subject compliance and attendance to the scheduled training sessions. None of the participating subjects in clinical treatment dropped out solely from the training intervention. Despite being simple and time-efficient to carry out, the training intervention likely benefits from supervision from a trained professional to provide commitment to, and understanding of, the training regime. This notion is also in agreement with previous studies employing training interventions in SUD-patients [33, 49]. Notably, our experience involves only patients participating in residential treatment. It should therefore be considered that the same feasibility and completion rates may not apply for outpatients.

\section{Maximal strength training and psychosocial health}

The SUD patients in the current study revealed significant signs of mood disorder at inclusion, reflected in elevated scores of anxiety and insomnia. The TG showed a reduction in both anxiety and insomnia scores, as well as a trend towards less depression. However, a reduction in depression following endurance training has previously been reported [33]. In combination, this is evidence that effective, intensive exercise training is not mentally harmful but, again, feasible. Since these improvements in this study are not significantly different from the CG it is difficult to conclude whether the mental health improvements were related to the clinical treatment itself or if they were a result of the improvements in muscle strength. Physical activity is in general shown to positively affect mental health [29], and it may therefore be that the mental health improvements are more related to physical activity performed by both groups, rather than the improvements in muscle strength. However, given the large beneficial effect of an improved physical capacity, and the substantial riskreduction for diseases and thus likely improvement in quality of life, a clinical treatment including effective physical training should be advocated. Indeed, a close association between physical training and mental health has previously been reported [50-52]. Furthermore, it should be questioned whether self-reporting questionnaires that are not able to detect large training-induced decreases in risk of lifestyle-related diseases are good enough.

\section{Clinical considerations for effective physical training in clinical treatment}

Recognizing the close relationship between physical capacities, life style related diseases and mortality $[10,53]$, it is likely that implementation of effective physical training as standard part of the treatment for SUD patients would decrease the high rates of non-drug related hospitalizations. This study shows that maximal strength training not only is feasible as a part of the treatment, it also has a large effect size and is time efficient. Previously we have shown similar findings for endurance training [33]. Adding to the arguments for implementation of effective physical training in the clinic is also the poor rehabilitation results observed in the CG participating in conventional physical activity. The current study observed that the muscular strength and force characteristics in the CG remained unchanged following the 8 week period. In a previous study similar observations were also reported for endurance capacity [33]. Although SUD patients suffer from many challenges, it is important to recognize that their physical health constitutes an important part of the overall health. Since muscle strength and aerobic capacity are known to be important contributors to the physical health, we would argue that strength- and endurance training should be carried out concurrently in clinical SUD treatment. Not only are these physical characteristics shown to be very low in SUD patients [6], today's treatment also appears to have very limited, if any endurance and strength effects. Importantly, this study, as well as a recent endurance training study [33] suggests that effective strengthand endurance training regimes are feasible and safe to carry out within this patient group.

Interestingly, the dropout rate in the TG (3 subjects) in the current study was lower than in the CG (5 subjects). Again, a similar finding was documented following endurance training ( 3 subjects) vs. conventional treatment (5 subjects) [33]. It is also of importance that the three subjects that dropped out of this study dropped out of the 
general clinical treatment, and not solely the adherence to the maximal strength training intervention. In support of this notion, it has previously been suggested that participation and adherence to an exercise program may have a positive effect on the relapse rates during alcohol recovery [32]. In combination, these findings suggest that implementation of effective physical training will improve the patients' physical health more than conventional treatment, and it is likely that it may also lead to gains in psychosocial health.

\section{Study limitations}

The training-induced changes of the main physiological variables were statistical significant in this study. However, a larger sample size may have been beneficial for the psychosocial variables, or perhaps a replacement by more detailed psychosocial questionnaires. While this study exemplifies that high intensity strength training is effective and feasible in SUD treatment, it should be noted that all patients in the current study had amphetamine as their primary drug, and that they were all recruited from the same clinic. While the conventional treatment in this clinic did not have any effect on the physical variables, it cannot be excluded that other clinics may have more effective treatment programs. Similarly, it can also be questioned whether our results would have been different if we had included patients with other primary drugs than amphetamine. As both patient characteristics and clinical treatment programs may vary between clinics, future studies should aim to investigate the effect of effective physical training in multiple clinics, and also aim for larger sample sizes to target psychosocial variables and include patients with different primary drugs.

\section{Conclusion}

This study shows that maximal strength training is a feasible, safe and effective method to improve muscle strength and function during SUD treatment. The large improvements in maximal strength and RFD that were observed following two months of training seemed to rely largely on neuromuscular adaptations. The improvements in physical health implies that the SUD patients have reduced their risk for traumas, falls and fractures, life style related diseases and all-cause mortality. Recognizing the poor physical condition of SUD patients, effective physical training, targeting muscle strength and aerobic capacity should be implemented in clinical treatment to improve physical and mental health.

\section{Abbreviations}

1RM: one repetition maximum; CG: control group; HAD: hospital anxiety \& depression scale; ISI: insomnia severity index; $M_{\text {max }}$ : maximal $M$-wave amplitude; MVC: maximal voluntary contraction; RFD: rate of force development;
SUD: substance use disorder; TG: training group; $V_{\text {max }}$ : maximal $V$-wave amplitude; V/M- ratio: maximal V-wave amplitude / maximal M-wave amplitude.

\section{Competing interests}

The authors declare that they have no competing interests.

\section{Authors' contributions}

$\mathrm{RU}$ has contributed as main author of the paper as well as physical testing. GF has contributed with subject recruitment, training and physical testing. $\mathrm{JH}$ has contributed with study design and writing of the paper. EW has contributed with study design and writing of the paper. All authors have read and approved the final version of the manuscript.

\section{Acknowledgements}

The authors would like to thank the subjects who volunteered to participate in this study for their time and efforts. The study was funded by the Norwegian University of Science and Technology.

\section{Author details}

${ }^{1}$ Department of Circulation and Medical imaging, Faculty of Medicine, the Norwegian University of Science and Technology, Prinsesse Kristinas gt. 3, 7006 Trondheim, Norway. ${ }^{2}$ Department of Research and Development, Clinic of Substance Use and Addiction Medicine, St. Olav University Hospital, Trondheim, Norway. ${ }^{3}$ Department of Physical Medicine and Rehabilitation, St. Olav University Hospital, Trondheim, Norway. ${ }^{4}$ Division of Psychiatry, Department of Østmarka, St. Olav University Hospital, Trondheim, Norway. ${ }^{5}$ Department of Internal Medicine, University of Utah, Salt Lake City, Utah, USA.

Received: 10 September 2015 Accepted: 29 February 2016

Published online: 31 March 2016

\section{References}

1. Nordentoft M, Wahlbeck K, Hallgren J, Westman J, Osby U, Alinaghizadeh H, et al. Excess mortality, causes of death and life expectancy in 270,770 patients with recent onset of mental disorders in Denmark, Finland and Sweden. PLoS One. 2013;8(1):e55176. doi:10.1371/journal.pone.0055176.

2. Stenbacka M, Leifman A, Romelsjo A. Mortality and cause of death among 1705 illicit drug users: a 37 year follow up. Drug Alcohol Rev. 2010;29(1):217. doi:10.1111/j.1465-3362.2009.00075.x.

3. Richards JR, Bretz SW, Johnson EB, Turnipseed SD, Brofeldt BT, Derlet RW. Methamphetamine abuse and emergency department utilization. West J Med. 1999:170(4):198-202.

4. Mosenthal AC, Livingston DH, Elcavage J, Merritt S, Stucker S. Falls: epidemiology and strategies for prevention. J Trauma. 1995:38(5):753-6.

5. Fang JF, Shih LY, Lin BC, Hsu YP. Pelvic fractures due to falls from a height in people with mental disorders. Injury. 2008;39(8):881-8. doi:10.1016/j.injury. 2008.03.012.

6. Flemmen $G$, Wang E. Impaired aerobic endurance and muscular strength in substance use disorder patients: implications for health and premature death. Med (Baltimore). 2015;94(44):e1914. doi:10.1097/MD. 0000000000001914

7. Pijnappels M, van der Burg PJ, Reeves ND, van Dieen JH. Identification of elderly fallers by muscle strength measures. Eur J Appl Physiol. 2008;102(5): 585-92. doi:10.1007/s00421-007-0613-6.

8. Jarvinen $T L$, Sievanen $H$, Khan KM, Heinonen A, Kannus P. Shifting the focus in fracture prevention from osteoporosis to falls. BMJ. 2008;336(7636):124-6. doi:10.1136/bmj.39428.470752.AD

9. Hoff J, Tjonna AE, Steinshamn S, Hoydal M, Richardson RS, Helgerud J. Maximal strength training of the legs in COPD: a therapy for mechanical inefficiency. Med Sci Sports Exerc. 2007;39(2):220-6. doi:10.1249/01.mss. 0000246989.48729.39.

10. Ruiz JR, Sui X, Lobelo F, Lee DC, Morrow Jr JR, Jackson AW, et al. Muscular strength and adiposity as predictors of adulthood cancer mortality in men. Cancer Epidemiol Biomarkers Prev. 2009;18(5):1468-76. doi:10.1158/10559965.EPI-08-1075.

11. Artero EG, Lee DC, Lavie CJ, Espana-Romero V, Sui X, Church TS, et al. Effects of muscular strength on cardiovascular risk factors and prognosis. J Cardiopulm Rehabil Prev. 2012·32(6):351-8. doi:10.1097/HCR. Ob013e3182642688. 
12. Ruiz JR, Sui X, Lobelo F, Morrow Jr JR, Jackson AW, Sjostrom M, et al. Association between muscular strength and mortality in men: prospective cohort study. BMJ. 2008;337:a439. doi:10.1136/bmj.a439.

13. Timpka S, Petersson IF, Zhou C, Englund M. Muscle strength in adolescent men and risk of cardiovascular disease events and mortality in middle age: a prospective cohort study. BMC Med. 2014;12(1):62. doi:10.1186/1741-7015-12-62.

14. Stenholm S, Mehta NK, Elo IT, Heliovaara M, Koskinen S, Aromaa A. Obesity and muscle strength as long-term determinants of all-cause mortality-a 33year follow-up of the Mini-Finland Health Examination Survey. Int J Obes (Lond). 2014;38(8):1126-32. doi:10.1038/ijo.2013.214.

15. Mosti MP, Kaehler N, Stunes AK, Hoff J, Syversen U. Maximal strength training in postmenopausal women with osteoporosis or osteopenia. J Strength Cond Res. 2013;27(10):2879-86. doi:10.1519/JSC. Ob013e318280d4e2.

16. Hill TR, Gjellesvik TI, Moen PM, Torhaug T, Fimland MS, Helgerud J, et al. Maximal strength training enhances strength and functional performance in chronic stroke survivors. Am J Phys Med Rehabil. 2012;91(5):393-400. doi:10. 1097/PHM.0b013e31824ad5b8.

17. Fimland MS, Helgerud J, Gruber M, Leivseth G, Hoff J. Enhanced neural drive after maximal strength training in multiple sclerosis patients. Eur J Appl Physiol. 2010;110(2):435-43. doi:10.1007/s00421-010-1519-2.

18. Wang E, Helgerud J, Loe H, Indseth K, Kaehler N, Hoff J. Maximal strength training improves walking performance in peripheral arterial disease patients. Scand J Med Sci Sports. 2010;20(5):764-70. doi:10.1111/j.1600-0838. 2009.01014.x.

19. Heggelund J, Morken G, Helgerud J, Nilsberg GE, Hoff J. Therapeutic effects of maximal strength training on walking efficiency in patients with schizophrenia - a pilot study. BMC Res Notes. 2012;5:344. doi:10.1186/17560500-5-344.

20. Storen O, Helgerud J, Stoa EM, Hoff J. Maximal strength training improves running economy in distance runners. Med Sci Sports Exerc. 2008;40(6): 1087-92. doi:10.1249/MSS.0b013e318168da2f.

21. Hvid L, Aagaard P, Justesen L, Bayer ML, Andersen JL, Ortenblad N, et al. Effects of aging on muscle mechanical function and muscle fiber morphology during short-term immobilization and subsequent retraining. J Appl Physiol (1985) 2010;109(6):1628-34. doi:10.1152/japplphysiol.00637.2010.

22. Wyszomierski SA, Chambers AJ, Cham R. Knee strength capabilities and slip severity. J Appl Biomech. 2009;25(2):140-8.

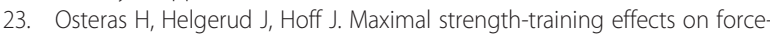
velocity and force-power relationships explain increases in aerobic performance in humans. Eur J Appl Physiol. 2002;88(3):255-63. doi:10.1007/ s00421-002-0717-y.

24. Aagaard P, Simonsen EB, Andersen JL, Magnusson P, Dyhre-Poulsen P. Increased rate of force development and neural drive of human skeletal muscle following resistance training. J Appl Physiol (1985). 2002;93(4):131826. doi:10.1152/japplphysiol.00283.2002.

25. Fimland MS, Helgerud J, Gruber M, Leivseth G, Hoff J. Functional maximal strength training induces neural transfer to single-joint tasks. Eur J Appl Physiol. 2009;107(1):21-9. doi:10.1007/s00421-009-1096-4.

26. Linke SE, Ussher M. Exercise-based treatments for substance use disorders: evidence, theory, and practicality. Am J Drug Alcohol Abuse. 2015;41(1):715. doi:10.3109/00952990.2014.976708.

27. Mamen A, Martinsen EW. Development of aerobic fitness of individuals with substance abuse/dependence following long-term individual physical activity. Eur J Sport Sci. 2010;10(4):255-62. doi:10.1080/17461390903377126.

28. Cassilhas RC, Antunes HK, Tufik S, de Mello MT. Mood, anxiety, and serum IGF-1 in elderly men given 24 weeks of high resistance exercise. Percept Mot Skills. 2010;110(1):265-76

29. Martinsen EW, Hoffart A, Solberg O. Comparing aerobic with nonaerobic forms of exercise in the treatment of clinical depression: a randomized trial. Compr Psychiatry. 1989;30(4):324-31.

30. Doyne EJ, Ossip-Klein DJ, Bowman ED, Osborn KM, McDougall-Wilson IB, Neimeyer RA. Running versus weight lifting in the treatment of depression. J Consult Clin Psychol. 1987;55(5):748-54.

31. Ortega FB, Silventoinen K, Tynelius P, Rasmussen F. Muscular strength in male adolescents and premature death: cohort study of one million participants. BMJ. 2012;345, e7279. doi:10.1136/bmj.e7279.

32. Brown RA, Abrantes AM, Read JP, Marcus BH, Jakicic J, Strong DR, et al. Aerobic exercise for alcohol recovery: rationale, program description, and preliminary findings. Behav Modif. 2009:33(2):220-49. doi:10.1177/ 0145445508329112.
33. Flemmen $G$, Unhjem R, Wang E. High-intensity interval training in patients with substance use disorder. Biomed Res Int. 2014;2014:616935. doi:10.1155/ 2014/616935.

34. McLellan AT, Kushner H, Metzger D, Peters R, Smith I, Grissom G, et al. The fifth edition of the addiction severity index. J Subst Abuse Treat. 1992;9(3):199-213.

35. Hermens HJ, Freriks B, Disselhorst-Klug C, Rau G. Development of recommendations for SEMG sensors and sensor placement procedures. J Electromyogr Kinesiol. 2000;10(5):361-74.

36. Bastien $\mathrm{CH}$, Vallieres $\mathrm{A}$, Morin $\mathrm{CM}$. Validation of the insomnia severity index as an outcome measure for insomnia research. Sleep Med. 2001;2(4):297307. doi:10.1016/\$1389-9457(00)00065-4.

37. Vaeroy H. Depression, anxiety, and history of substance abuse among Norwegian inmates in preventive detention: reasons to worry? BMC Psychiatry. 2011;11:40. doi:10.1186/1471-244X-11-40.

38. Helgerud J, Karlsen T, Kim WY, Hoydal KL, Stoylen A, Pedersen H, et al. Interval and strength training in CAD patients. Int J Sports Med. 2011;32(1): 54-9. doi:10.1055/s-0030-1267180.

39. Karlsen T, Helgerud J, Stoylen A, Lauritsen N, Hoff J. Maximal strength training restores walking mechanical efficiency in heart patients. Int J Sports Med. 2009;30(5):337-42. doi:10.1055/s-0028-1105946.

40. Moreland JD, Richardson JA, Goldsmith $\mathrm{CH}$, Clase CM. Muscle weakness and falls in older adults: a systematic review and meta-analysis. J Am Geriatr Soc. 2004;52(7):1121-9. doi:10.1111/j.1532-5415.2004.52310.x.

41. Skelton DA, Kennedy J, Rutherford OM. Explosive power and asymmetry in leg muscle function in frequent fallers and non-fallers aged over 65. Age Ageing. 2002;31(2):119-25.

42. Sale DG, Martin JE, Moroz DE. Hypertrophy without increased isometric strength after weight training. Eur J Appl Physiol Occup Physiol. 1992;64(1):51-5.

43. Behm DG, Sale DG. Intended rather than actual movement velocity determines velocity-specific training response. J Appl Physiol (1985). 1993; 74(1):359-68.

44. Aagaard P, Simonsen EB, Andersen JL, Magnusson P, Dyhre-Poulsen P. Neural adaptation to resistance training: changes in evoked $\mathrm{V}$-wave and $\mathrm{H}$ reflex responses. J Appl Physiol (1985). 2002;92(6):2309-18. doi:10.1152/ japplphysiol.01185.2001.

45. Del Balso C, Cafarelli E. Adaptations in the activation of human skeletal muscle induced by short-term isometric resistance training. J Appl Physiol (1985). 2007;103(1):402-11. doi:10.1152/japplphysiol.00477.2006.

46. Vila-Cha C, Falla D, Correia MV, Farina D. Changes in $H$ reflex and $V$ wave following short-term endurance and strength training. J Appl Physiol (1985). 2012;112(1):54-63. doi:10.1152/japplphysiol.00802.2011.

47. Sparr LF, Moffitt MC, Ward MF. Missed psychiatric appointments - who returns and who stays away. Am J Psychiat. 1993;150(5):801-5.

48. Ball SA, Carroll KM, Canning-Ball M, Rounsaville BJ. Reasons for dropout from drug abuse treatment: symptoms, personality, and motivation. Addict Behav. 2006;31(2):320-30. doi:10.1016/j.addbeh.2005.05.013.

49. Mamen A, Pallesen S, Martinsen EW. Changes in mental distress following individualized physical training in patients suffering from chemical dependence. Eur J Sport Sci. 2011;11(4):269-76. doi:10.1080/ 17461391.2010.509889.

50. Martinsen EW, Medhus A, Sandvik L. Effects of aerobic exercise on depression - a controlled-study. Brit Med J. 1985;291(6488):109.

51. Galper DI, Trivedi MH, Barlow CE, Dunn AL, Kampert JB. Inverse association between physical inactivity and mental health in men and women. Med Sci Sport Exer. 2006;38(1):173-8. doi:10.1249/01.mss.0000180883.32116.28.

52. Mota-Pereira J, Silverio J, Carvalho S, Ribeiro JC, Fonte D, Ramos J. Moderate exercise improves depression parameters in treatment-resistant patients with major depressive disorder. J Psychiatr Res. 2011;45(8):1005-11. doi:10. 1016/j.jpsychires.2011.02.005.

53. Myers J, Prakash M, Froelicher V, Do D, Partington S, Atwood JE. Exercise capacity and mortality among men referred for exercise testing. N Engl J Med. 2002;346(11):793-801. doi:10.1056/NEJMoa011858. 